\title{
Fulminant Hepatic Failure and Fatal Cerebral Edema Following Clostridium perfringens Bacteremia: Case Report and Review of Literature
}

\author{
Alice Shen ${ }^{1}$, Gabriel O. Ologun ${ }^{1}$, Robert Behm ${ }^{2}$ \\ 1. General Surgery, Guthrie Clinic/Robert Packer Hospital 2. Trauma/Critical Care, Guthrie Clinic/Robert \\ Packer Hospital
}

$\square$ Corresponding author: Alice Shen, shihyuan73@gmail.com

Disclosures can be found in Additional Information at the end of the article

\section{Abstract}

Clostridium perfringens (CP) bacteremia is a rare but rapidly fatal infection. Only 36 cases of CP bacteremia with gas containing liver abscesses on image studies have been reported in the literature since 1990. In this report, we describe a 65-year-old diabetic male with CP bacteremia which progressed into fulminant hepatic failure with subsequent fatal cerebral edema.

Categories: Radiology, General Surgery, Infectious Disease

Keywords: clostridium perfringens, fulminant liver failure, hepatic abscesses, fatal cerebral edema, diabetes mellitus

\section{Introduction}

Clostridium perfringens (CP) bacteremia has been described as a rare and rapidly fatal disease secondary to massive hemolytic anemia, resulting in progressive multi-organ failure. The mortality rate exceeds $80 \%$ despite high-dose intravenous antibiotics and source control [1]. It is commonly associated with diabetes, malignancy, and immunosuppression [2]. In this report, we present a 65-year-old diabetic male with CP bacteremia which progressed into fulminant hepatic failure with subsequent fatal cerebral edema.

\section{Case Presentation}

A 65-year-old male with a history of diabetes mellitus and coronary artery disease presented to an outside hospital emergency department with an one-day history of abdominal pain. He was febrile to $101.3^{\circ} \mathrm{F}$ with leukocytosis of $32 \mathrm{~K} / \mu \mathrm{L}$, elevated transaminases and hyperbilirubinemia (total bilirubin of $7.8 \mathrm{mg} / \mathrm{dL}$ ). The working diagnosis was cholangitis. The patient was started on broad-spectrum intravenous antibiotics, and transferred to our hospital for endoscopic retrograde cholangiopancreatogram (ERCP) and further management.

The ERCP revealed no evidence of biliary duct obstruction (Figure 1), and a non-obstructing biliary stone was found in a duodenal diverticulum associated with the major papilla (Figure 2). A computed tomography (CT) scan of the abdomen and pelvis was obtained which showed multiple hepatic abscesses containing air fluid levels involving the right hepatic lobe. These abscesses were not amenable to percutaneous drainage (Figure 3). Due to concerns of gaseous organism infection, repeat ERCP with sphincterotomy and stent placement were performed with the intention of source control. Blood culture from the outside hospital returned as 


\section{Cureus}

Clostridium perfringens.

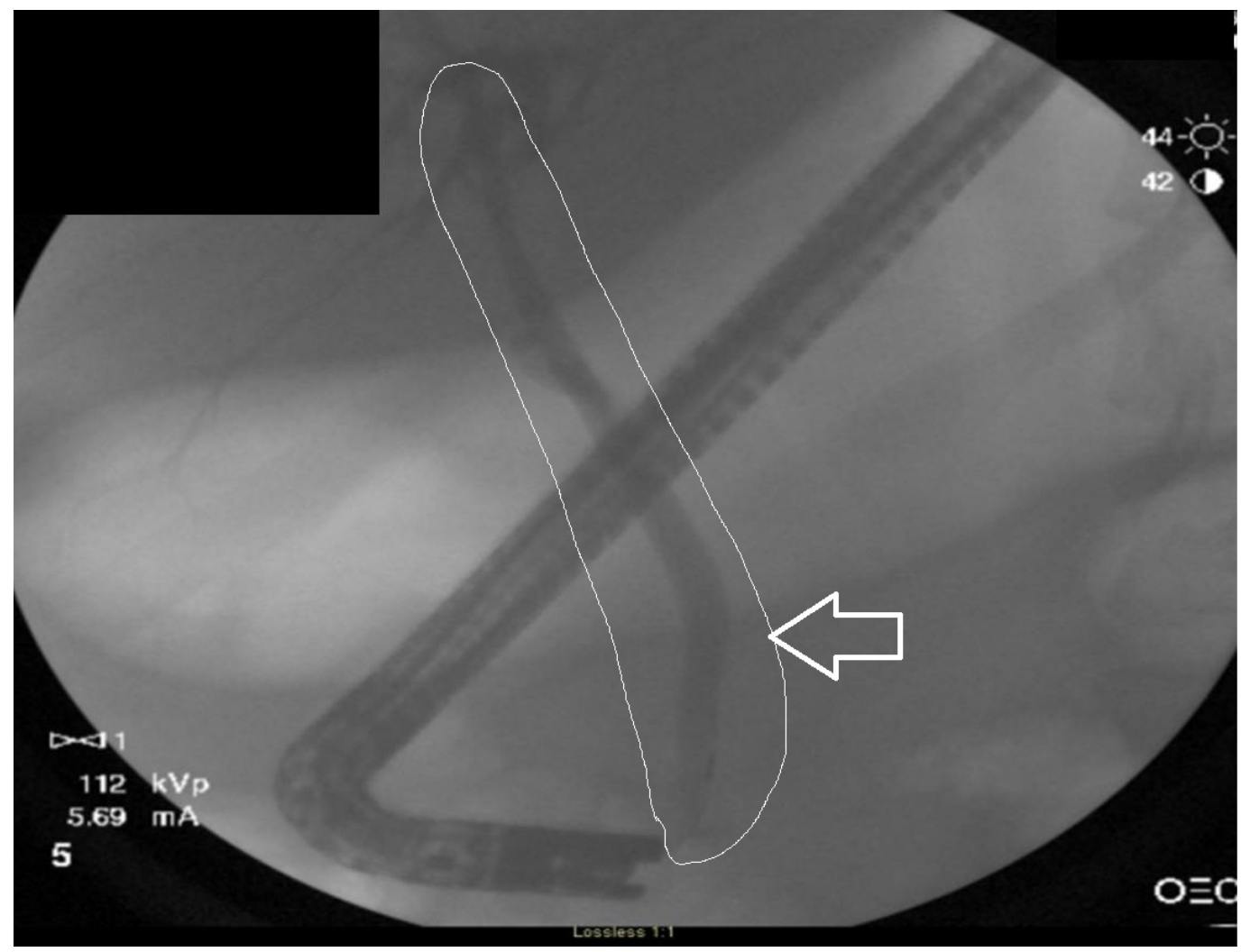

FIGURE 1: The first endoscopic retrograde cholangiopancreatogram (ERCP) revealing absence of filling defect in the biliary tree (outlined area and arrow). 


\section{Cureus}

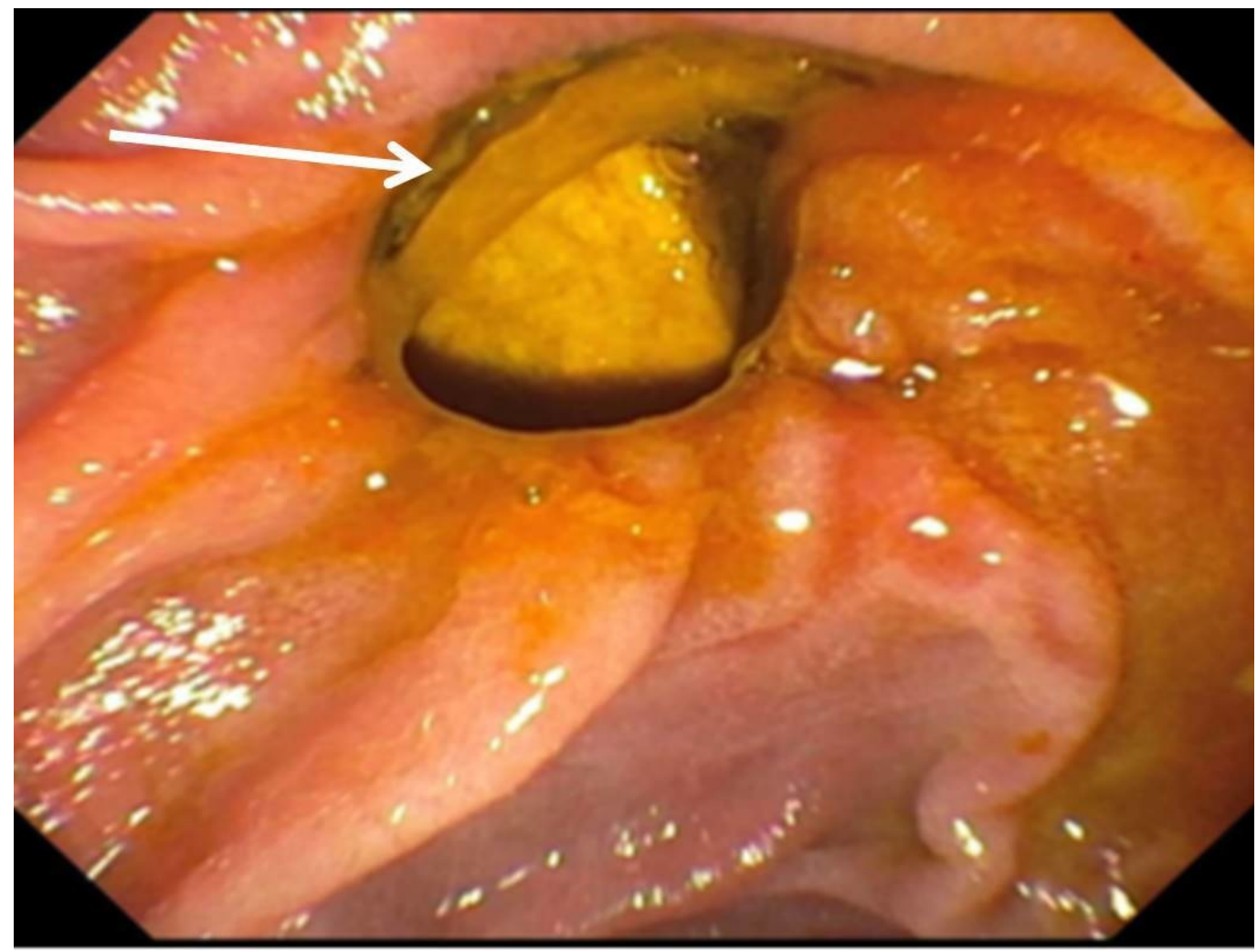

FIGURE 2: Duodenal diverticulum containing stone.

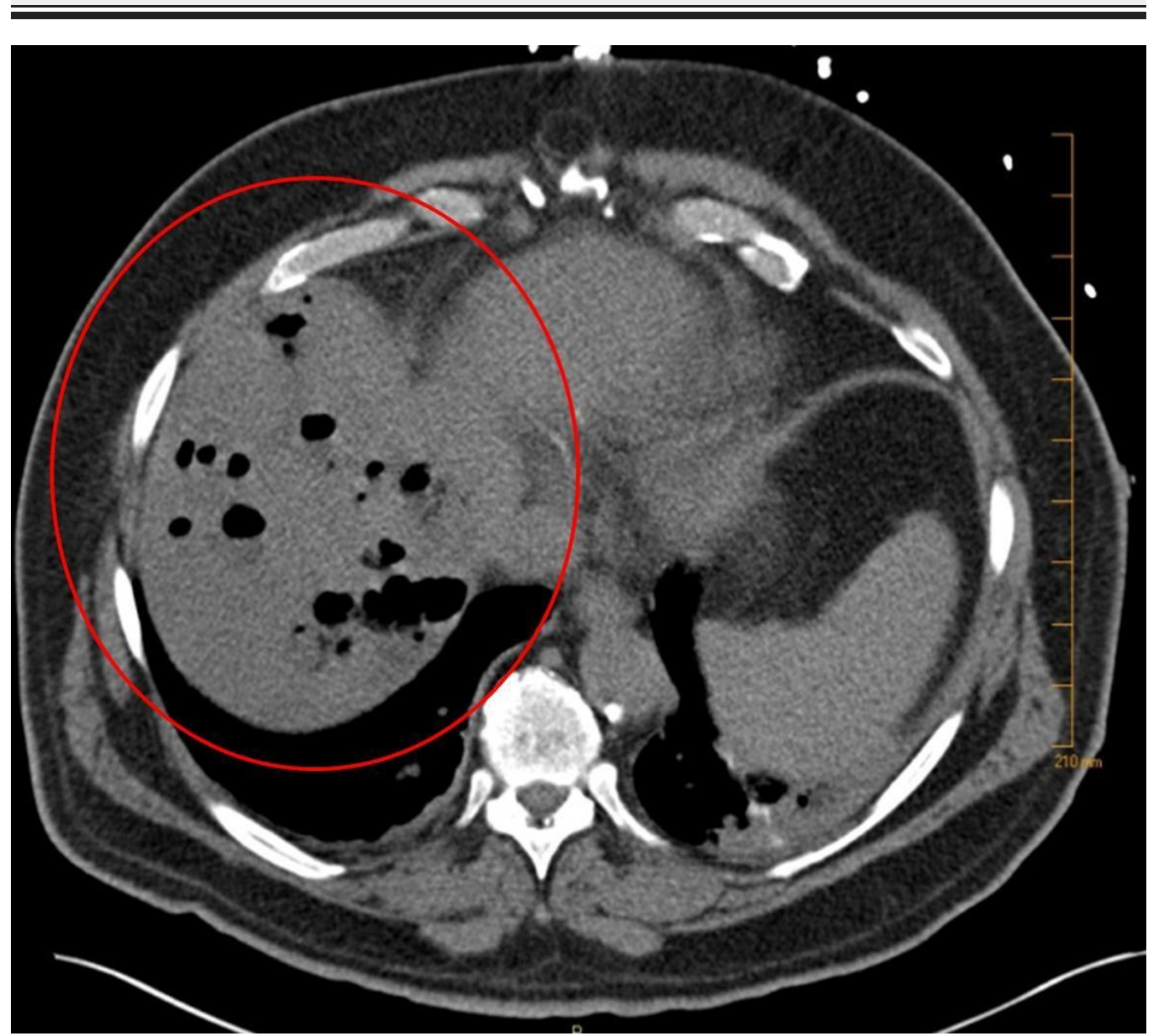




\section{Cureus}

\section{FIGURE 3: Multiple hepatic abscesses and multiple air fluid levels.}

On hospital day four, general surgery was consulted. Repeat CT scans of the abdomen showed no worsening of the liver abscesses. However, the patient continued to decline clinically, with sepsis progressing to multi-system organ failure, requiring hemodialysis. Furthermore, he developed an altered mental status and was intubated for airway protection. Given his worsening clinical picture, the patient was taken to the operating room where he underwent open surgical debridement and drainage with ultrasound guidance. The CT scan obtained on postoperative day two demonstrated resolution of liver abscesses (Figure 4). The blood cultures obtained in our institution were negative. However, the hepatic abscess culture grew Saccharomyces cerevisiae (SC) after 10 days of incubation.

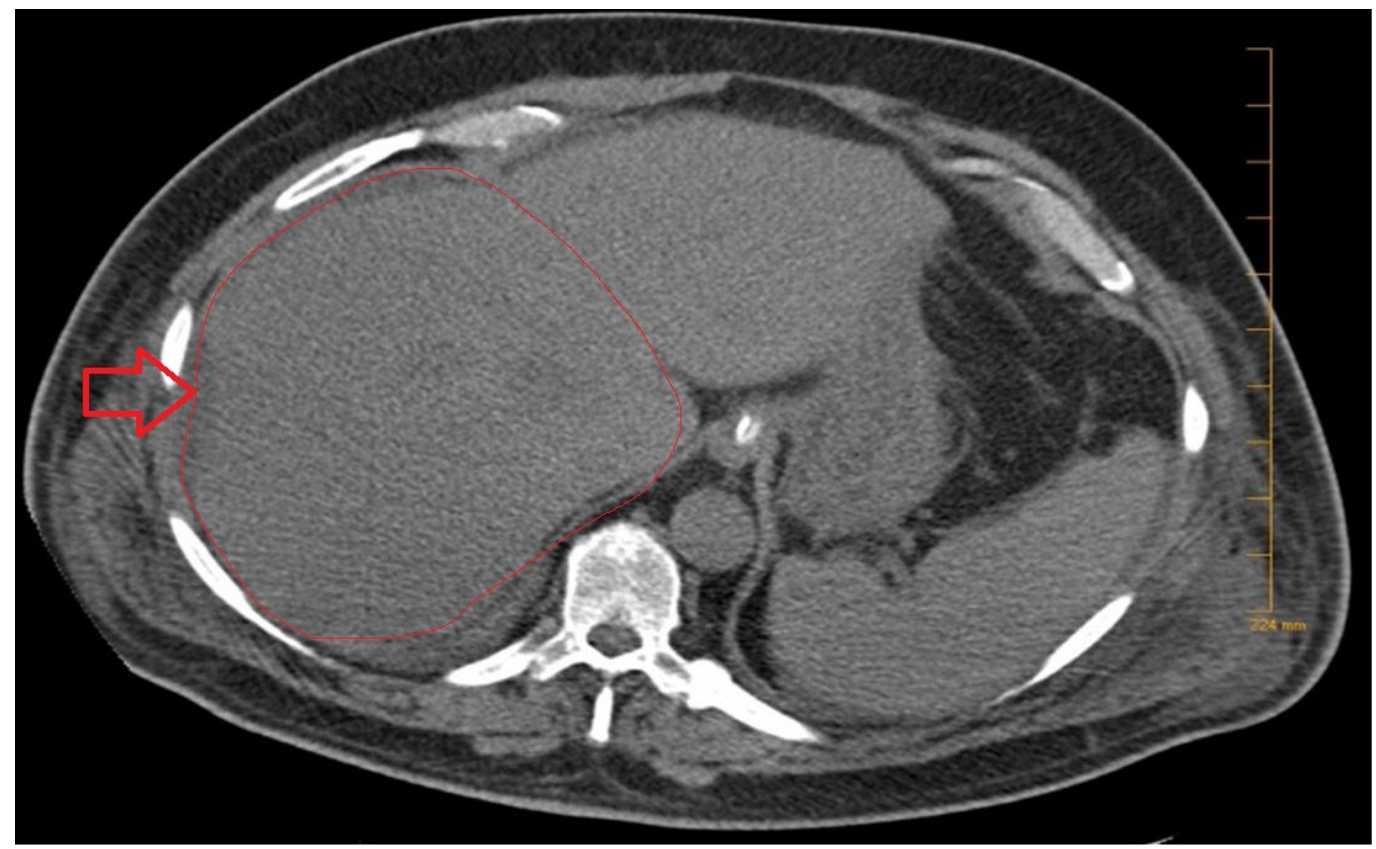

\section{FIGURE 4: Resolution of liver abscess status post-surgical drainage. Outline and arrow indicating area of interest.}

On postoperative day three, the patient had a seizure episode, prompting a CT scan of the head which demonstrated cerebral edema. Magnetic resonance imaging (MRI-angiogram) of the head was done, revealing no vascular abnormality. The cerebral edema was thought to be secondary to acute liver failure. A repeat CT scan of the head, on postoperative day five revealed progressive diffuse cerebral edema with the development of obstructive hydrocephalus (Figure 5). Neurosurgery was consulted, who felt further intervention would not alleviate an impending herniation. On hospital day eleven, the patient's family opted for comfort care measures. He was terminally extubated, expiring shortly thereafter. 


\section{Cureus}

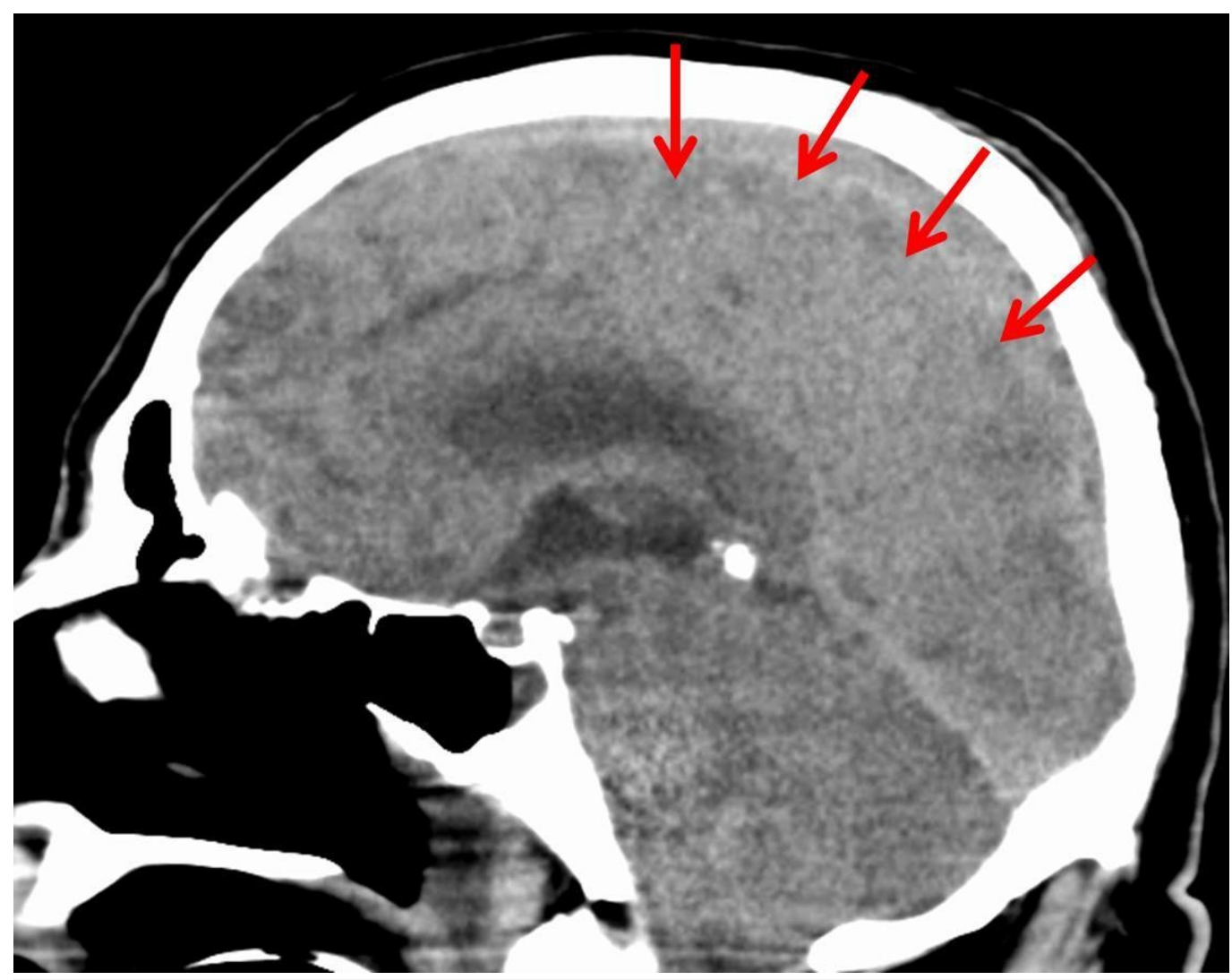

FIGURE 5: Severe and diffuse cerebral edema with obstructive hydrocephalus.

\section{Discussion}

Law and Lee [3] reviewed 20 cases of CP bacteremia with liver abscesses from 1990 to 2012, showing a median time from admission to death of 11 hours. In contrast to those described in the literature, our patient did not have massive intravascular hemolysis, hypotension on presentation, or a rapidly fatal outcome. Although his initial blood cultures were positive for CP at outside hospital, his repeat blood cultures in our hospital were negative for organisms. This negative result could be explained by early initiation of intravenous antibiotics.

The patient's hepatic culture data was significant for the late growth of Saccharomyces cerevisiae. One case of liver abscess positive for SC was described in 1990 [4]. In this review, CP was also isolated in the liver abscess culture but the blood culture was positive for Pseudomonas aeruginosa. This discordance of microorganism between blood culture and liver abscess cultures was also described in other cases of CP abscess [5-6].

It is unclear whether this patient truly had CP liver abscess because hepatic fluid failed to isolate CP. Nevertheless, he presented with clinical features of pyogenic liver abscess: fever, epigastric pain, bacteremia, and the presence of gas on liver image studies. The liver culture could be false negative for $\mathrm{CP}$. There was also a case in the literature reported with positive $\mathrm{CP}$ blood culture with failure to isolate CP from liver abscess culture [7].

We proposed that his fulminant hepatic failure was the primary event leading to his clinical outcome. This would explain the lack of CP in the liver abscess culture. The majority of fulminant liver failure are idiopathic. The hepatic cell death and necrosis, as well as the ERCP 
performed prior to the CT scan, could contribute to the hepatic gas shown on the CT image. The abscess formation pattern during the debridement was noted to be in the hepatic vein distribution, which has been shown to be more consistent with liver necrosis rather than a liver abscess. This could explain the patient's lack of overwhelming shock expected with CP bacteremia. To our knowledge, this is the first documented case of CP bacteremia associated with fulminant liver failure which progressed to fatal cerebral edema.

We reviewed 37 cases of CP bacteremia with associated liver abscesses reported since 1990 (Table 1). These cases had an average age of 67 years (range: 42 to 84 ; 26 patients were male (70\%), and 11 were female (30\%). The most common underlying condition was diabetes (41\%). There were eight patients with diabetes as the only medical co-morbidity. Ten patients (27\%) had malignancy. Two (5\%) had a history of transplantation for primary sclerosing cholangitis or alcohol cirrhosis who were on immunosuppressive therapy. Eight (20\%) had a history of hepatobiliary pancreatic operations or procedures within two months prior the diagnosis of CP liver abscesses. Six (16\%) had no underlying conditions.

\begin{tabular}{|c|c|c|c|c|c|c|c|c|c|c|c|c|c|}
\hline No. & Author & Year & Age & Sex & Underlying Conditions(s) & WBC & $\mathrm{Hb}$ & TBili & BCX & $\begin{array}{l}\text { Hepatic } \\
\text { drain }\end{array}$ & $\begin{array}{l}\text { Liver } \\
\text { Cx }\end{array}$ & $\begin{array}{l}\text { Survi- } \\
\text { val }\end{array}$ & $\begin{array}{l}\text { Hrs } \\
\text { present. } \\
\text { to } \\
\text { death }\end{array}$ \\
\hline 1 & Batge [7] & 1992 & 61 & M & $\begin{array}{l}\text { DM, pancreatic cancer s/p } \\
\text { Whipple }\end{array}$ & 38.2 & 11.6 & 43.9 & + & Yes - p & - & Yes & N/A \\
\hline 2 & Rogstad & 1993 & 61 & M & None & $\mathrm{N} / \mathrm{A}$ & 16.7 & $\mathrm{~N} / \mathrm{A}$ & + & No & $+(\mathrm{a})$ & No & 3 \\
\hline 3 & Gutierrez & 1995 & 14 & M & None & 19.8 & 13.1 & 4.1 & + & No & $+(\mathrm{a})$ & No & 6 \\
\hline 4 & Jones & 1996 & 66 & $F$ & Liver Transplant & 11.3 & 11.3 & 4.4 & + & No & $+(\mathrm{a})$ & No & 10 \\
\hline 5 & Eckel & 2000 & 65 & $\mathbf{F}$ & CBD CA meta s/p TACE & 13 & 11.2 & 4.6 & + & Yes - p & $\mathrm{nr}$ & Yes & N/A \\
\hline 6 & Kreidl & 2002 & 80 & M & DM, ESRD on HD & 29 & 10.7 & 12.6 & + & No & $+(\mathrm{a})$ & No & 11 \\
\hline 7 & Pichon & 2003 & 42 & $\mathbf{F}$ & Alcohol cirrhosis & 6.2 & 10.2 & 12.3 & + & No & $\mathrm{nr}$ & Yes & N/A \\
\hline 8 & Quigley & 2003 & 73 & M & Ischemic heart & 11 & 14.2 & 4.2 & $\mathrm{nr}$ & No & $+(\mathrm{a})$ & No & $\begin{array}{l}\text { Died at } \\
\text { home }\end{array}$ \\
\hline 9 & Bergert [5] & 2004 & 58 & M & $\begin{array}{l}\text { Pancreatic cancer s/p } \\
\text { Whipple } 1 \text { yr }\end{array}$ & 34.1 & N/A & 7.7 & $\begin{array}{l}\text { E. } \\
\text { coli }\end{array}$ & Yes - p & + & No & 24 \\
\hline 10 & $\mathrm{Au}$ & 2005 & 65 & M & DM, ESRD on HD & 25 & 6.2 & 9.4 & $\mathrm{nr}$ & No & + & No & 72 \\
\hline 11 & Fondran & 2005 & 63 & M & $\begin{array}{l}\text { pancreatic cancer meta to } \\
\text { liver }\end{array}$ & N/A & N/A & N/A & + & Yes - O & $\mathrm{nr}$ & Yes & N/A \\
\hline 12 & Daly & 2006 & 80 & M & DM & N/A & 8.7 & N/A & + & No & $\mathrm{nr}$ & No & 3 \\
\hline 13 & Ohfani & 2006 & 78 & M & DM & 18.6 & 10 & 1.4 & + & No & $+(\mathrm{a})$ & No & 3 \\
\hline 14 & Loran & 2006 & 69 & M & None & 26 & 8.7 & N/A & $\mathrm{nr}$ & No & $+(a)$ & No & 6 \\
\hline 15 & $\begin{array}{l}\text { Alarcon Del } \\
\text { Agua }\end{array}$ & 2009 & 14 & M & Stroke & 16 & N/A & 3.7 & + & Yes - O & + & Yes & N/A \\
\hline
\end{tabular}




\section{Cureus}

\begin{tabular}{|c|c|c|c|c|c|c|c|c|c|c|c|c|c|}
\hline 16 & Merino & 2009 & 83 & $\mathbf{F}$ & None & 26.5 & 12.2 & 19.6 & + & No & $\mathrm{nr}$ & No & 72 \\
\hline 17 & Meyns & 2009 & 64 & M & $\begin{array}{l}\text { DM, myelodysplastic } \\
\text { syndrome }\end{array}$ & 1.5 & 7.2 & 8.3 & + & Yes - p & + & No & 48 \\
\hline 18 & Tabarelli & 2009 & 65 & $\mathbf{F}$ & $\begin{array}{l}\text { pancreatic cancer s/p } \\
\text { Whipple }\end{array}$ & N/A & N/A & N/A & + & Yes - p & + & No & 120 \\
\hline 19 & Bradly [2] & 2010 & 52 & M & HCC s/p RY HJ & 11.6 & N/A & 17.4 & + & No & $+(\mathrm{a})$ & No & 6 \\
\hline 20 & $\mathrm{Ng}$ & 2010 & 61 & $\mathbf{F}$ & DM & 16.8 & 13.5 & 15.4 & + & Yes - O & $\mathrm{nr}$ & Yes & N/A \\
\hline 21 & Rajendran & 2010 & 58 & M & None & 14.6 & 13.3 & N/A & + & Yes - O & + & Yes & N/A \\
\hline 22 & Law [3] & 2012 & 50 & $\mathbf{F}$ & Rectal Cancer & 46.3 & 8.3 & 8.9 & + & Yes - p & $\mathrm{nr}$ & No & 168 \\
\hline 23 & Qandeel & 2012 & 59 & $\mathbf{F}$ & DM, s/p cholecystectomy & N/A & 9.1 & N/A & + & Yes - O & $\mathrm{nr}$ & Yes & N/A \\
\hline 24 & $\begin{array}{l}\text { Sathiyam- } \\
\text { oorthy }\end{array}$ & 2012 & 65 & $\mathbf{F}$ & DM & N/A & N/A & N/A & + & Yes - p & $\mathrm{nr}$ & Yes & N/A \\
\hline 25 & Imai & 2014 & 76 & M & HTN & 33.2 & 12.2 & 10.5 & + & Yes - p & + & No & 5 \\
\hline 26 & Kitterer & 2014 & 71 & M & Liver Transplant & 18.5 & 11.2 & 3.7 & + & Yes - O & $\mathrm{nr}$ & No & 13 \\
\hline 27 & Kurasawa & 2014 & 65 & M & DM & 24.8 & 13.5 & 6.4 & + & No & $\mathrm{nr}$ & No & 6 \\
\hline 28 & Cochrane & 2015 & 65 & $\mathbf{F}$ & DM & normal & 13 & 9.6 & + & Yes - p & $\mathrm{nr}$ & Yes & N/A \\
\hline 29 & Eltawansy & 2015 & 81 & $\mathbf{F}$ & Stroke & 22 & N/A & 0.4 & + & Yes - p & + & No & 120 \\
\hline 30 & Khan & 2015 & 77 & $\mathrm{M}$ & DM, HTN & 33 & 6 & 9.5 & - & No & $+(\mathrm{a})$ & No & 7 \\
\hline 31 & $\mathrm{Li}$ & 2015 & 71 & $\mathrm{M}$ & HCC s/p TACE & 11.5 & 11.8 & 18.9 & + & No & $\mathrm{nr}$ & Yes & N/A \\
\hline 32 & Rives & 2015 & 63 & M & $\begin{array}{l}\text { Colon CA meta to liver. ERCP } \\
\text { for obs. Jaundice }\end{array}$ & 21.3 & N/A & 7.6 & + & Yes - p & $\mathrm{nr}$ & Yes & N/A \\
\hline 33 & Garcia & 2016 & 65 & $M$ & DM, Chiari I & 22.2 & 14.6 & 2.6 & + & Yes - p & + & Yes & N/A \\
\hline 34 & Hashiba & 2016 & 82 & $\mathrm{M}$ & DM & 30.1 & 8.3 & 9 & + & No & $\mathrm{nr}$ & No & 2 \\
\hline 35 & Kyang [6] & 2016 & 84 & M & $\begin{array}{l}\text { gastric cancer meta to liver } \\
\text { s/p MW ablasion }\end{array}$ & 4 & N/A & N/A & $\begin{array}{l}\text { E. } \\
\text { coli }\end{array}$ & Yes - p & + & Yes & N/A \\
\hline 36 & Lim & 2016 & 58 & M & None & 14.2 & 12.4 & N/A & + & No & $\mathrm{nr}$ & No & 7.5 \\
\hline 37 & Shen & 2017 & 65 & M & DM & 32 & 12 & 7.8 & + & Yes - O & S. c. & No & 264 \\
\hline
\end{tabular}

\section{TABLE 1: Cases of Clostridium perfringens infection associated with gas-containing}

liver abscesses on image studies published since 1990.

$\mathrm{Hb}=$ reported hemoglobin (g/dL); WBC = white blood cell count $(\mathrm{K}-\mathrm{g} / \mathrm{L})$; TBili = total bilirubin $(\mathrm{mg} / \mathrm{dL})$; $\mathrm{LDH}=\mathrm{Lactate}$ dehydrogenase; $\mathrm{M}$ = male; $\mathrm{F}$ = female; $\mathrm{DM}$ = diabetes mellitus; $\mathrm{CBD}=$ common bile duct; $\mathrm{CA}$ = cancer; $\mathrm{TACE}=$ transarterial chemoembolization; ESRD = end-stage renal disease; HD = hemodialysis; $\mathrm{HCC}=$ hepatocellular carcinoma; RY HJ = Roux-en-Y hepatojejunostomy; $\mathrm{MW}$ = microwave; Yes- $\mathrm{p}=$ percutaneous drainage; Yes- $\mathrm{O}=$ operative drainage; $\mathrm{BCx}=$ blood culture; Liver Cx= Liver Culture; + = positive for Clostridium perfringens; $\mathrm{nr}=$ not reported or not obtained; $\mathrm{E}$. coli = positive for $\mathrm{E}$. coli but negative for Clostridium perfringens; - = negative for C. perfringens; (a) = autopsy; S.c.= positive for Saccharomyces cerevisiae, negative for Clostridium perfringens. Hrs = hours; N/A = does not apply. 


\section{Cureus}

The relationship of the blood and liver abscess culture results was also evaluated. CP infection was diagnosed on the basis of positive blood or liver abscess culture results, although only 13 cases (35\%) had definitive concordance between the two culture results. Nine cases (25\%) of positive liver abscess cultures were from the autopsy. Fifteen (41\%) cases did not report or did not obtain liver abscess culture results.

The survival rate is only $37.8 \%$ among the reviewed cases. Of the 14 survivors, 12 (85\%) had the liver abscesses removed. The median time from presentation to death for mortality is 10.3 hours, which is similar to the previous report of 11 hours [3].

\section{Conclusions}

$\mathrm{CP}$ bacteremia remains a rare but life-threatening disease which requires timely diagnosis, early systemic antibiotics, and source control. A timely diagnosis could be a challenge because of the time required for the blood culture result. A clinical picture of fever, epigastric pain, bacteremia, and hepatic gas-formation on imaging studies should prompt the diagnosis of CP infection. For associated liver abscesses, either percutaneous drainage or surgical drainage is acceptable. For patients who present with acute liver failure, timely referral to a transplant center is imperative to improve the chance of survival.

\section{Additional Information}

\section{Disclosures}

Human subjects: Consent was obtained by all participants in this study. Conflicts of interest: In compliance with the ICMJE uniform disclosure form, all authors declare the following: Payment/services info: All authors have declared that no financial support was received from any organization for the submitted work. Financial relationships: All authors have declared that they have no financial relationships at present or within the previous three years with any organizations that might have an interest in the submitted work. Other relationships: All authors have declared that there are no other relationships or activities that could appear to have influenced the submitted work.

\section{Acknowledgements}

The authors would like to thank Thomas J. Vandermeer and Burt Cagir for reviewing the manuscript.

\section{References}

1. van Bunderen CC, Bomers MK, Wesdorp E, et al: Clostridium perfringens septicaemia with massive intravascular haemolysis: a case report and review of the literature. Neth J Med. 2010, 68:343-6.

2. Bradly DP, Collier M, Frankel J, et al.: Acute necrotizing cholangiohepatitis with Clostridium perfringens: a rare cause of post-transplantation mortality. Gastroenterol Hepatol (N Y). 2010, 6(4):241-3.

3. Law ST, Lee MK: A middle-aged lady with a pyogenic liver abscess caused by clostridium perfringens. World J Hepatol. 2012, 4(8):252-5. 10.4254/wjh.v4.i8.252

4. Aucott JN, Fayen J, Grossnicklas H, et al.: Invasive infection with saccharomyces cerevisiae: report of three cases and review. Rev Infect Dis. 1990, 12(3):406-11. 10.1093/clinids/12.3.406

5. Bergert $\mathrm{H}$, Illert $\mathrm{T}$, Friedrich $\mathrm{K}$, et al.: Fulminant liver failure following infection by Clostridium perfringens. Surg Infect (Larchmt). 2004, 5(2):205-9. 10.1089/sur.2004.5.205 


\section{Cureus}

6. Kyang LS, Bin Traiki TA, Alzahrani NA, et al.: Microwave ablation of liver metastasis complicated by Clostridium perfringens gas-forming pyogenic liver abscess (GPLA) in a patient with past gastrectomy. Int J Surg Case Rep. 2016, 27:32-35.

10.1016/j.ijscr.2016.08.009

7. Batge B, Filejski W, Kurowski V, et al.: Clostridial sepsis with massive intravascular hemolysis: rapid diagnosis and successful treatment. Intensive Care Med. 1992, 18(8):488-90.

10.1007\%2FBF01708587?LI=true 